\title{
Trabectedin verzögert die Krankheitsprogression
}

Die Therapie mit Trabectedin kann bei Patientinnen mit partiell platinsensitivem Ovarialkarzinom mit einem platinfreien Intervall von mehr als 6 Monaten das progressionfreie und das Gesamtüberleben verlängern.

Die ursprünglich aus der Seescheide Ecteinascidia turbinata gewonnene Substanz Trabectedin sorgt für einen deutlichen Fortschritt bei der Behandlung des Ovarialkarzinoms. Das belegen die Ergebnisse der Phase-III-Studie OVA-301 mit Patientinnen mit platinsensitivem oder -resistentem Ovarialkarzinomrezidiv. Durch die Kombination von pegyliertem liposomalem Doxorubicin (PLD) mit Trabectedin wurde ein im Vergleich zur alleinigen PLD-Gabe signifikant verlänger-

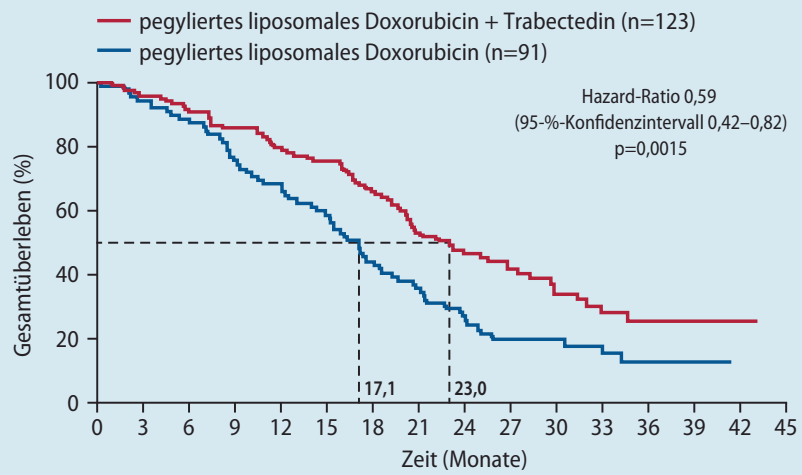

nach Poveda A et al., 2010, Ann Oncol: Epub ahead of print

OVA-301-Studie - Gesamtüberleben der Patientinnen mit partiell platinsensitivem Ovarialkarzinomrezidiv: pegyliertes liposomales Doxorubicin (PLD) plus Trabectedin versus PLD allein tes progressionsfreies Überleben (PFS) erreicht (median 7,3 Monate versus 5,8 Monate; $p=0,0190$ ). Es resultierte damit eine etwa $20 \%$ ige Reduktion der Progression unter PLD plus Trabectedin (Hazard-Ratio [HR] 0,79; 95-\%-Konfidenzintervall [95-\%-KI] 0,65-0,96).

Besondere Vorteile bei partiell platinsensitivem Ovarialkarzinom Insbesondere profitierten dabei Patientinnen mit platinsensitivem Ovarialkarzinomrezidiv. Besonders günstig waren die Ergebnisse aber auch in der Subgruppe der Patientinnen mit partiell platinsensitivem Ovarialkarzinomrezidiv und einem platinfreien Intervall von mehr als 6 Monaten, so Jochen Schütte, Düsseldorf. Das mediane PFS wurde durch die zusätzliche Gabe von Trabectedin in dieser Subgruppe von 5,5 Monaten unter PLD allein auf 7,4 Monate verlängert ( $\mathrm{HR} 0,65$; 95-\%-KI 0,45-0,92; p=0,0152; Poveda A et al., 2010, Ann Oncol: Epub ahead of print). Das Gesamtüberleben stieg signifikant von 17,1 Monaten unter PLD allein auf 23,0 Monate unter der Kombination von PLD mit Trabectedin ( $p=0,0015 ;$ Abb.).

Trabectedin zeichnet sich laut Schütte durch ein gutes Verträglichkeitsprofil aus, was sich unter anderem daran zeigt, dass die Lebensqualität der Patientinnen in beiden Studienarmen, das heißt unter PLD allein und unter der Kombination von PLD mit Trabectedin, vergleichbar war. Eine kumulative Toxizität besteht nicht, ebenfalls keine Neuro- oder Kardiotoxizität. CV

Quelle: Veranstaltung von Pharma Mar S.A.

\section{Mobilisation peripherer Blutstammzellen}

\section{Biosimilar vergleichbar effektiv}

Die Ergebnisse retrospektiver Untersuchungen mit kleinen Fallzahlen weisen darauf hin, dass das Filgrastim-Biosimilar Ratiograstim ${ }^{\circledast}$ ebenso effektiv in der Mobilisierung peripherer Blutstammzellen (PBPC) ist wie das Referenzprodukt.

Das Biosimilar Ratiograstim ${ }^{\circledast}$ ist ein rekombinant hergestellter Granulozyten-koloniestimulierender Faktor (G-CSF), dessen Wirksamkeit in der Behandlung chemotherapiebedingter Neutropenie sich in 3 Phase-IIIStudien als vergleichbar mit der des Referenzprodukts Neupogen ${ }^{\circledast}$ erwiesen hatte (Del Giglio A et al., 2008, BMC Cancer 8: 332; Engert A et al., 2009, Leuk Lymphoma 50: 374-379; Gatzemeier U et al., 2009, J Thorac
Oncol 4: 736-740). „Allerdings gibt es bisher nur wenige klinische Daten zum Einsatz des Biosimilars für die Mobilisation von PBPC", erklärte Amy Publicover, Southampton/ Großbritannien. Deshalb verglichen Publicover und Kollegen retrospektiv die Daten von 29 Patienten, die im Rahmen einer autologen Stammzelltransplantation das Biosimilar erhalten hatten, mit den Daten von 34 Patienten, deren PBPC mit dem StandardG-CSF mobilisiert worden waren. „Die Untersuchung zeigte keine Unterschiede zwischen dem Biosimilar und der Kontrolle", so Publicover. Auch Hartmut Kirchner, Hannover, stellte retrospektiv fest, dass in seinem heterogenen Kollektiv von 20 Patienten beim Einsatz des Biosimilars eine ausrei- chende Stammzellmenge mobilisiert werden konnte. In einer kleinen Untersuchung zur allogenen Transplantation mit 10 Patienten und 8 Spendern zeigte sich das Biosimilar laut Michael Schmitt, Rostock, ebenfalls gleichwertig mit dem Standard.

\section{Besondere Nebenwirkungen bislang nicht beobachtet}

Besondere Nebenwirkungen, die über die bekannten unspezifischen Knochenbeschwerden während der Regenerationsphase hinausgingen, wurden in keiner der 3 Untersuchungen bemerkt. Gleichwohl liegen Beobachtungen zu Langzeitwirkungen von G-CSF noch nicht vor. „Bisher gibt es allerdings noch keinen Hinweis darauf, dass G-CSF die Entwicklung von Tumoren begünstigt", sagte Hans-Jochem Kolb, München. SG

Quelle: Veranstaltung von ratiopharm direct 